\title{
Mapping breast cancer blood flow index, composition, and metabolism in a human subject using combined diffuse optical spectroscopic imaging and diffuse correlation spectroscopy
}

Hossein S. Yazdi

Thomas D. O'Sullivan

Anais Leproux

Brian Hill

Amanda Durkin

Seraphim Telep

Jesse Lam

Siavash S. Yazdi

Alice M. Police

Robert M. Carroll

Freddie J. Combs

Tomas Strömberg

Arjun G. Yodh

Bruce J. Tromberg 


\title{
Mapping breast cancer blood flow index, composition, and metabolism in a human subject using combined diffuse optical spectroscopic imaging and diffuse correlation spectroscopy
}

\author{
Hossein S. Yazdi, ${ }^{a}$ Thomas D. O'Sullivan, ${ }^{a}$ Anais Leproux, ${ }^{a}$ Brian Hill, ${ }^{a}$ Amanda Durkin, ${ }^{\text {a }}$ Seraphim Telep, ${ }^{a}$ \\ Jesse Lam, ${ }^{a}$ Siavash S. Yazdi, ${ }^{a}$ Alice M. Police, ${ }^{b}$ Robert M. Carroll, ${ }^{b}$ Freddie J. Combs, ${ }^{b}$ Tomas Strömberg, ${ }^{\text {a,c }}$ \\ Arjun G. Yodh, ${ }^{\mathrm{d}}$ and Bruce J. Tromberg ${ }^{\mathrm{a}, \star}$ \\ aUniversity of California, Beckman Laser Institute and Medical Clinic, Irvine, California, United States \\ bUniversity of California, Chao Family Comprehensive Cancer Center, Orange, California, United States \\ 'Linköping University, Department of Biomedical Engineering, Linköping, Sweden \\ dUniversity of Pennsylvania, Department of Physics and Astronomy, Philadelphia, Pennsylvania, United States
}

\begin{abstract}
Diffuse optical spectroscopic imaging (DOSI) and diffuse correlation spectroscopy (DCS) are modelbased near-infrared (NIR) methods that measure tissue optical properties (broadband absorption, $\mu_{\mathrm{a}}$, and reduced scattering, $\mu_{\mathrm{s}}^{\prime}$ ) and blood flow (blood flow index, BFI), respectively. DOSI-derived $\mu_{\mathrm{a}}$ values are used to determine composition by calculating the tissue concentration of oxy- and deoxyhemoglobin $\left(\mathrm{HbO}_{2}\right.$, $\mathrm{HbR}$ ), water, and lipid. We developed and evaluated a combined, coregistered DOSI/DCS handheld probe for mapping and imaging these parameters. We show that uncertainties of $0.3 \mathrm{~mm}^{-1}(37 \%)$ in $\mu_{\mathrm{s}}^{\prime}$ and $0.003 \mathrm{~mm}^{-1}(33 \%)$ in $\mu_{\mathrm{a}}$ lead to $\sim 53 \%$ and $9 \%$ errors in BFI, respectively. DOSI/DCS imaging of a solid tissue-simulating flow phantom and a breast cancer patient reveals well-defined spatial distributions of BFI and composition that clearly delineates both the flow channel and the tumor. BFI reconstructed with DOSI-corrected $\mu_{\mathrm{a}}$ and $\mu_{\mathrm{s}}^{\prime}$ values had a tumor/normal contrast of $2.7,50 \%$ higher than the contrast using commonly assumed fixed optical properties. In conclusion, spatially coregistered imaging of DOSI and DCS enhances intrinsic tumor contrast and information content. This is particularly important for imaging diseased tissues where there are significant spatial variations in $\mu_{\mathrm{a}}$ and $\mu_{\mathrm{s}}^{\prime}$ as well as potential uncoupling between flow and metabolism. ( ) The Authors. Published by SPIE under a Creative Commons Attribution 3.0 Unported License. Distribution or reproduction of this work in whole or in part requires full attribution of the original publication, including its DOI. [DOI: 10.1117/1.JBO.22.4.045003]
\end{abstract}

Keywords: noninvasive; breast cancer; diffuse optical spectroscopy; oxygen metabolism; blood flow; diffuse correlation spectroscopy. Paper 160803R received Nov. 23, 2016; accepted for publication Mar. 13, 2017; published online Apr. 5, 2017.

\section{Introduction}

Diffuse optical spectroscopic imaging (DOSI) is a functional imaging technology that measures hemodynamics and biochemical composition in centimeter-thick tissues. ${ }^{1}$ We have developed a broadband handheld scanning probe for imaging breast tumors under clinically relevant conditions, such as detecting tumors in mammographically dense tissue, ${ }^{2}$ distinguishing between malignant and benign tumors, ${ }^{3}$ monitoring tumor response ${ }^{4-6}$ to chemotherapy, and assessing breast density. ${ }^{7}$ In these studies, broadband NIR data (650 to $1000 \mathrm{~nm}$ ) are used to calculate tissue absorption and scattering spectra and develop various metrics of endogenous contrast based primarily on tissue concentrations of oxy- and deoxyhemoglobin $\left(\mathrm{HbO}_{2}\right.$, $\mathrm{HbR}$ ), water, and lipid. To enhance this information and provide a more complete picture of biochemical composition and oxygen consumption inside and in the vicinity of breast lesions, DOSI can be combined with diffuse correlation spectroscopy (DCS), a complementary method that measures deep tissue blood flow.

DCS utilizes the fluctuations of detected light intensity from the movement of particles to probe microvasculature blood flow

*Address all correspondence to: Bruce J. Tromberg, E-mail: bjtrombe@ @ci.edu in deep tissue. ${ }^{8-10}$ The blood flow index (BFI) measured by DCS has been validated by arterial spin-labeled magnetic resonance imaging, ${ }^{11}$ Doppler ultrasound, ${ }^{12,13}$ Xenon computed tomography, ${ }^{14}$ and laser Doppler flowmetry. ${ }^{15,16}$ Further, DCS systems have been used to monitor blood flow changes in clinical applications associated with brain, ${ }^{13,14,17}$ skeletal muscle, ${ }^{11,18,19}$ and tumors. ${ }^{10,20-23}$ When taken together, DCS and DOSI can provide a noninvasive measurement of the metabolic rate of oxygen consumption in tissue, and indeed, several studies have shown that the combination of DOSI and DCS techniques in breast lesion characterization provides a better understanding of tumor physiology and metabolism and may enhance treatment monitoring in the presurgical setting. ${ }^{20,23,24}$ However, these breast lesion studies were performed sequentially by using separate DCS and DOSI instruments. Serial measurements add additional time for data collection and can lead to uncertainties in the origins of the different signals.

This study aims to develop and validate a spatially coregistered, DOSI/DCS metabolic imaging system for enhancing breast tumor characterization. We combined DCS and DOSI techniques into a single handheld probe and developed integrated software that collects the data nearly simultaneously. Since the BFI depends on tissue optical properties, the BFI is typically calculated using fixed values from literature or using 
values measured with a separate instrument. DOSI is unique because it provides these optical properties (absorption, $\mu_{\mathrm{a}}$, and reduced scattering, $\mu_{\mathrm{s}}^{\prime}$, parameters) over a broad spectral range from 650 to $1000 \mathrm{~nm}^{25}$ The impact of $\mu_{\mathrm{a}}$ and $\mu_{\mathrm{s}}^{\prime}$ on BFI has been investigated in a previous study using a homogeneous liquid phantom where particles undergo Brownian motion. ${ }^{26}$ We have extended this work to more realistic tissue simulating phantoms that employ a buried flow channel designed to simulate a subsurface flowing inhomogeneity. This allows us to analyze, for the first time, the combined impact of optical properties $\left(\mu_{\mathrm{a}}, \mu_{\mathrm{s}}^{\prime}\right)$, flow velocity, and channel depth on the estimation of the BFI.

Coregistered DOSI and DCS were then applied to construct two-dimensional (2-D) maps of subsurface particle flow in a solid tissue-simulating phantom with an embedded flow channel. Finally, images of tissue BFI, composition, and relative metabolism were obtained, for the first time, by coregistered DOSI/DCS mapping in a clinical measurement of a breast cancer patient. DOSI-derived optical properties at each measurement point were used to correct BFI in both clinical and tissue phantom images, improving accuracy and revealing unique and complementary contrast in breast cancer.

\section{Material and Methods}

\subsection{DCS Theory}

A typical DCS setup using optical fibers in tissue contact for light source and detector is shown in Fig. 1(a). The light source utilizes a long coherence length laser. The backscattered light intensity $I(t)$ is detected at a distance $\rho$ from the source. The temporal speckle fluctuations of $I(t)$ is analyzed by calculating the normalized light intensity temporal autocorrelation function $\left(g_{2}\right): g_{2}(\tau)=\frac{\langle I(t) I(t+\tau)\rangle}{\langle I(t)\rangle^{2}}$, where $\tau$ represents the correlation delay time. Temporal speckle fluctuations cause variations in detected light intensity; thus, motions of red blood cells in microvascular blood vessels (arterioles, capillaries, and venules) provide the dominant contrast mechanism. ${ }^{8,11,27,28}$ Speckle fluctuations from a medium with higher flow rate produce a faster decay of $g_{2}$. The Siegert equation ${ }^{29}$ relates the normalized electric field autotemporal correlation function, $g_{1}(\tau)$, to $g_{2}(\tau)$

$g_{2}(\tau)=1+\beta\left|g_{1}(\tau)\right|^{2}$ (a)

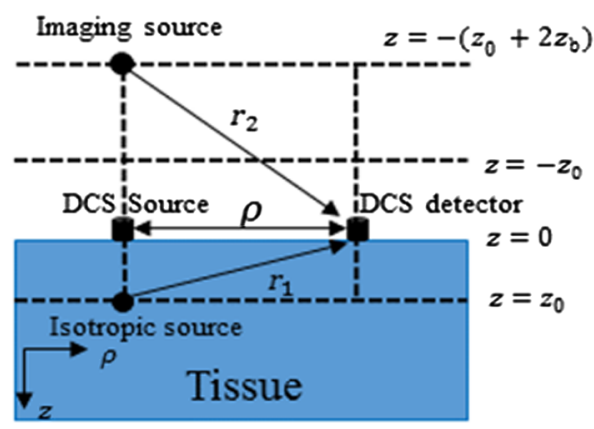

(c)

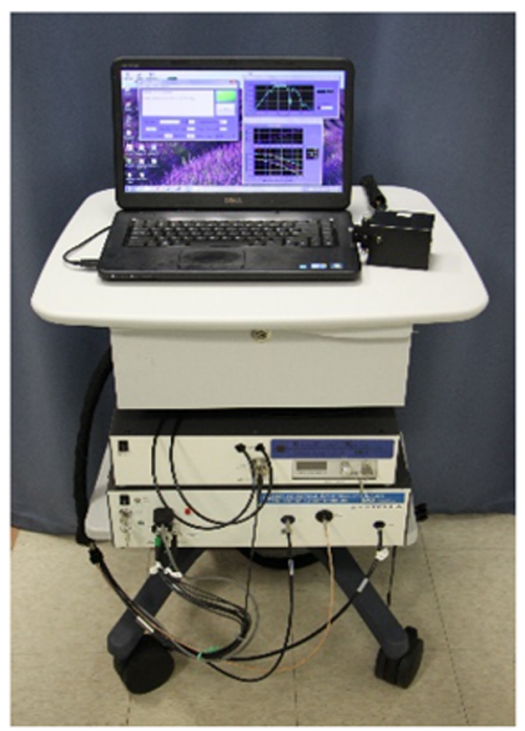

(b)

(d)
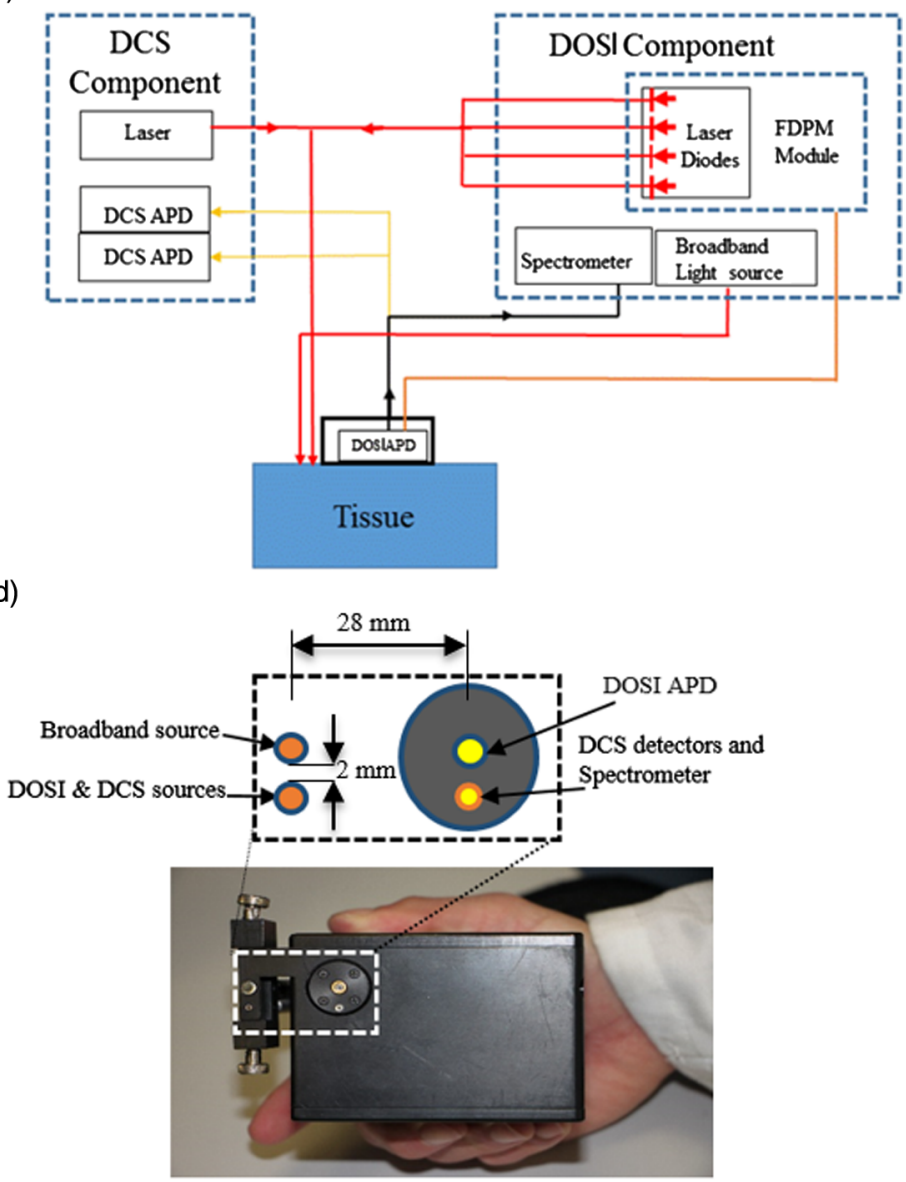

Fig. 1 (a) The semi-infinite measurement geometry for DCS including a positive isotropic light sources and a negative isotropic light (imaging) source for DCS theory; see Sec. 2.1 and Ref. 30 for details. (b) Block diagram of the DOSI/DCS instrument, red color represents the multimode source fibers. Collection fiber bundle is shown by black, which contains a DOSI spectrometer fiber and two DCS single mode fibers (shown with yellow), and orange is an RF cable. (c) Photograph of the entire system on a portable cart and (d) diagram and photograph of handheld probe. 
where $\beta$ depends on the optical setup and can be experimentally determined. ${ }^{30}$

Similar to the photon diffusion equation, a correlation diffusion equation that describes the propagation of the unnormalized electric field temporal autocorrelation function $G_{1}(\tau)$ in a turbid media can be derived. For the setup in Fig. 1(a), ${ }^{8,9,15,30-33}$

$G_{1}(\rho, z, \tau)=\frac{3 \mu_{\mathrm{s}}^{\prime}}{4 \pi}\left[\frac{e^{-k(\tau) r_{1}}}{r_{1}}-\frac{e^{-k(\tau) r_{2}}}{r_{2}}\right]$,

where $\quad r_{1}=\left[\rho^{2}+\left(z-z_{0}\right)^{2}\right]^{\frac{1}{2}}, \quad r_{2}=\left[\rho^{2}+\left(z+z_{0}+2 z_{b}\right)^{2}\right]^{\frac{1}{2}}$, $z_{0}=\frac{1}{\mu_{\mathrm{s}}^{\prime}}, \quad z_{b}=\frac{2\left(1+R_{\text {eff }}\right)}{3 \mu_{\mathrm{s}}^{\prime}\left(1-R_{\mathrm{eff}}\right)}, \quad$ and $\quad R_{\text {eff }}=-1.440 n^{-2}+0.71 n^{-1}+$ $0.668+0.0636 n$ is the effective reflection coefficient, which is determined by the ratio of the refraction indices of two medium (e.g., $n=\frac{n_{\text {tissue }}}{n_{\text {air }}} \approx 1.33$ ). Equation (2) incorporates the extrapolated zero boundary condition, which approximates the isotropic light source located at $z=z_{0}$ and negative isotropic imaging source located at $z=-\left(z_{0}+2 z_{b}\right)$. Schematic of the extrapolated boundary geometry is shown in Fig. 1(a). ${ }^{30,32-34}$ The effective decay rate $k(\tau)$ of $G_{1}(\rho, \tau)$ provides information about the motion of the scatterers in the underlying medium: $k^{2}(\tau)=3 \mu_{\mathrm{s}}^{\prime} \mu_{\mathrm{a}}+\mu_{\mathrm{s}}^{\prime 2} k_{0}^{2} \alpha\left\langle\Delta r^{2}(\tau)\right\rangle$ where $\left\langle\Delta r^{2}(\tau)\right\rangle$ is the mean square displacement of the moving particles, $k_{0}$ is the wave-vector magnitude of the incident light field, and $\alpha$ is proportional to the fraction of scatterers that are moving (i.e., the fraction of tissue scatterers that are red blood cells). For diffusive motion $\left\langle\Delta r^{2}(\tau)\right\rangle=6 D_{b} \tau$, where $D_{b}$ is the effective Brownian diffusion coefficient for the tissue scatterers. ${ }^{30}$ The BFI $\left(\mathrm{mm}^{2} / \mathrm{s}\right)$ is defined as BFI $=\alpha D_{b} \cdot{ }^{15,35}$ We used the tissue optical properties measured with DOSI at each measurement iteration to calculate BFI. Assuming $\alpha=1$, the factor $k(\tau)$ is fitted to the measured autocorrelation function, $g_{2}(\rho, \tau)$, via Eqs. (1) and (2) to derive the BFI. ${ }^{15}$

\subsection{Instrumentation}

A block diagram illustrating the combined DOSI and DCS system is shown in Fig. 1(b). The entire system and controlling PC fits on a cart for easy transport in a clinical setting [Fig. 1(c)].

\subsubsection{DOSI hardware}

DOSI estimates broadband near-infrared (NIR) (650 to $1000 \mathrm{~nm})$ reduced scattering $\left(\mu_{\mathrm{s}}^{\prime}\right)$ and absorption coefficients $\left(\mu_{\mathrm{a}}\right)$ of turbid media through a combination of frequency domain photon migration (FDPM) and continuous wave (CW) NIR spectroscopy. ${ }^{25}$ The principle of the measurement has been described $;^{36}$ the system utilized here is based on a custom $500-\mathrm{MHz}$ frequency-domain module ${ }^{37}$ and a high-speed 1mm-diameter avalanche photodiode (APD) detector (\#C5658 with S6045-03 APD, Hamamatsu Photonics, Shizuoka Pref., Japan). Four laser diodes $(660,690,780$, and 830) are sequentially swept from 50 to $500 \mathrm{MHz}$ (each $20 \mathrm{~mW}$ average power at the tissue) to acquire multifrequency phase and amplitude measurements of diffusely propagating photon density waves for each wavelength. The CW component of the DOSI system consists of a tungsten-halogen white light source (Micropak HL2000-HP, Ocean Optics, Florida) and a spectrometer (B\&W Tek, Inc. Model 611, Delaware) to measure the backscattered reflectance spectra in the wavelength range 650 to $1000 \mathrm{~nm}$.

\subsubsection{DCS hardware}

The DCS optical source is a long coherence-length $(>10 \mathrm{~m})$ CW 785-nm laser (Crystalaser, Nevada). Laser output was adjusted to $40 \mathrm{~mW}$, which was determined to ensure a safe intensity with reasonable detected photon count rate (more than 60 kcounts/s) for all the measurements. The DCS detectors consisted of two photon-counting modules (SPCM-AQRH15-FC, Excelitas, Canada). The digital output of each detector was connected to a four-channel hardware correlator board (Flex01LQ-5, correlator.com, New Jersey) to measure the autocorrelation function $\left(g_{2}\right)$ of the detected signal. Dark counts due to ambient light leakage were reduced by placing the detector units in an optical isolation box inside the system chassis.

\subsubsection{Integrated DOSI/DCS handheld probe}

DOSI and DCS sources and detectors were integrated into a single handheld probe with variable source-detector (s-d) separations $(22,28$, or $34 \mathrm{~mm})$ [Fig. 1(d)]. The s-d separations were set to $28 \mathrm{~mm}$ for all data reported here. Light sources for the DCS measurements and the FDPM component of DOSI were delivered with a five-fiber bundle of $200 \mu \mathrm{m}$ step-index multimode fibers. The CW light source component of DOSI was delivered with a 3-mm-diameter bundle of $50-\mu \mathrm{m}$ multimode fibers. The detector fibers include two single mode fibers (DCS detectors) and a 1-mm-diameter solid, multimode fiber (DOSI broadband detector/spectrometer) (LEONI Fiber Optics Inc., Virginia). The temperature-controlled FDPM APD detector was built into the probe for direct contact with the tissue. Custom software controls the serial collection of DOSI and DCS data and allows the instrument operator to easily enable either or both techniques. This feature allowed us to select the combined approach in the region of interest to minimize impact on the overall data collection time.

\subsection{Liquid Phantom Experiment}

The system was characterized using an liquid intralipid (IL)based system with a submerged flow tube (3 mm ID, $0.8 \mathrm{~mm}$ wall), which allowed us to dynamically vary flow tube IL and background IL optical properties, flow rate, and depth of the flow tube. The combined (DOSI/DCS) handheld probe was wrapped with thin plastic film and secured at the surface of the liquid. FDPM calibration was done with the plastic wrap to factor out the effect of the plastic on the measurement.

A mixture of IL and nigrosin ink was used as a moving liquid through the flow tube for all the experiments. In each case, the predicted $\mu_{\mathrm{s}}^{\prime}$ and $\mu_{\mathrm{a}}$ of the moving liquid at $785 \mathrm{~nm}$ were 20 and $0.06 \mathrm{~mm}^{-1}$, respectively. These values are determined based on DOSI measurements. Three individual experiments were performed to study the effect of depth, absorption, and scattering on BFI: (1) to study the effect of depth and flow rates on BFI, the liquid surrounding the tube was formulated to simulate breast tissue $\left(\mu_{\mathrm{a}}=0.005 \mathrm{~mm}^{-1}\right.$ and $\left.\mu_{\mathrm{s}}^{\prime}=0.8 \mathrm{~mm}^{-1}\right)$ and distance between top of the flow tube, and probe surface (depth) varied from 0 to $20 \mathrm{~mm}$ in five steps (5 mm increment in each step). The flow rate varied between 0 and $400 \mathrm{~mL} / \mathrm{min}$ in each depth. $g_{2}$ data samples were taken at each flow rate, and DCS integration time was $5 \mathrm{~s}$ for measuring $g_{2}$. The relative change of blood flow, $\mathrm{rBF}$, is the ratio of measured BFI for each flow rate and $\mathrm{BFI}$ for zero flow ( $\left.\mathrm{rBF}=\frac{\mathrm{BFI}_{\text {flow }}}{\mathrm{BFI}_{\text {zero flow }}}\right)$. (2) To study the effect of $\mu_{\mathrm{a}}$ 
on BFI, the absorption of the background liquid was changed in 10 steps from 0.0035 to $0.015 \mathrm{~mm}^{-1}$ with constant $\mu_{\mathrm{s}}^{\prime}$ presented as mean $(\mathrm{M}) \pm$ standard deviation $(\mathrm{SD})$ was $0.74 \pm 0.031 \mathrm{~mm}^{-1}$. The flow tube was set at $10 \mathrm{~mm}$ depth and a constant $25 \mathrm{~mL} / \mathrm{min}$ flow rate. (3) To study the effect of $\mu_{\mathrm{s}}^{\prime}$ on BFI, the scattering of the background liquid was changed in nine steps from 0.43 to $1.67 \mathrm{~mm}^{-1}$ at a constant $\mu_{\mathrm{a}}\left(0.0041 \pm 0.00027 \mathrm{~mm}^{-1}\right)$. Similar to the previous experiment, DOSI and DCS measurements were taken at each step with a constant $25 \mathrm{~mL} /$ min pump flow rate.

\subsection{Silicone Phantom with Flow Channel}

To demonstrate the capability of the probe for providing coregistered DOSI and DCS imaging, a solid phantom was made with a 4.6-mm-diameter flow channel, the top of which was located $5 \mathrm{~mm}$ below the surface. The phantom was created with $2.7 \mathrm{~mL}$ nigrosin $(0.015 \%), 1.05 \mathrm{mg}$ titanium dioxide $(0.88 \mathrm{~g} / \mathrm{L})$, and $1.2 \mathrm{~L}$ polydimethylsiloxane, according to Ref. 11 . The previously described moving IL mixture was used inside the flow channel.

\subsection{Human Subject Measurement}

The integrated system was used to characterize a triple negative (ER-, PR-, Her2-), grade 3 infiltrating ductal carcinoma of a 28year-old premenopausal patient. The patient was measured 3 days following her first neoadjuvant chemotherapy infusion. The $20 \times 30 \mathrm{~mm}$ tumor was located $16 \mathrm{~mm}$ below the surface, as measured by ultrasound. The subject provided written informed consent, and the study protocol was approved by the University of California Institutional Review Board (UCI HS\# 1995-563).

Tissue chromophore concentrations $\mathrm{HbR}, \mathrm{HbO}_{2}$, total hemoglobin (THb), lipid, water, and the tissue optical index (TOI $\left.=\frac{[\mathrm{HbR}] \times[\text { water }]}{[\text { lipid }]}\right)$ were calculated from the broadband absorption spectrum. The TOI is a composite index that reveals contrast in more metabolically active regions (i.e., tumor) and was developed to identify breast tumor location from DOSI data. ${ }^{7,36}$ In addition, the relative tumor-to-normal oxygen consumption in mammary tissue $\left(\mathrm{rMMRO}_{2(\mathrm{~T} / \mathrm{N})}\right)$ was calculated by combining DOSI and DCS data using ${ }^{23}$

$$
\operatorname{rMMRO}_{2\left(\frac{T}{N}\right)}=\frac{\gamma_{T}}{\gamma_{N}} \cdot\left(\frac{\mathrm{HbR}_{T}}{\mathrm{HbR}_{N}}\right) \cdot\left(\frac{\mathrm{THb}_{T}}{\mathrm{THb}_{N}}\right)^{-1} \cdot\left(\frac{\mathrm{BFI}_{T}}{\mathrm{BFI}_{N}}\right)
$$

where $T$ and $N$ represent values from tumor and normal breast tissue locations, respectively. In this model, $\gamma_{T(N)}$ for either tumor or normal is the ratio of $\mathrm{HbR}$ to $\mathrm{THb}$ concentration in venous vasculature compare to the ratio of $\mathrm{HbR}$ to $\mathrm{THb}$ in the total vasculature. The ratio of $\frac{\gamma_{T}}{\gamma_{N}}$ was assumed to be 1 for simplicity. Further details can be found in Ref. 23. Normal tissue values of each parameter ( $\mathrm{HbR}, \mathrm{THb}$, and $\mathrm{BFI}$ ) were calculated using an average of three points far from the tumor region.

\subsection{Image Analysis}

The surface of the solid phantom was measured over a $90 \mathrm{~mm} \times$ $90 \mathrm{~mm}$ field of view in $5 \mathrm{~mm}$ increments, centered on the flow tube. The resulting $19 \times 19$ grid consisted of a total of 361 measurement points. Coregistered DOSI/DCS scans were obtained at each point, and images were generated using cubic interpolation between the measured points. The in vivo study was performed using a $90 \mathrm{~mm} \times 80 \mathrm{~mm}$ field of view in $10 \mathrm{~mm}$ increments that covered the tumor and surrounding normal breast tissue. The resulting $9 \times 8$ grid consisted of 72 DOSI measurement points. To minimize overall measurement time, combined DOSI/DCS data were acquired over a smaller $30 \times 80 \mathrm{~mm}$ field of view using a $3 \times 8$ grid of 24 measurement points in $10 \mathrm{~mm}$ increments centered over the tumor. Similar to the silicon phantom study, functional maps of tissue chromophore concentrations $\left[\mathrm{HbR}, \mathrm{HbO}_{2}\right.$, total hemoglobin (THb), lipid, water, TOI] and BFI were reconstructed from each grid point location. Map points were interpolated using the 2-D cubic interpolation function (interp2) in MATLAB ${ }^{\circledR}$ to round out the discrete shapes. (a)

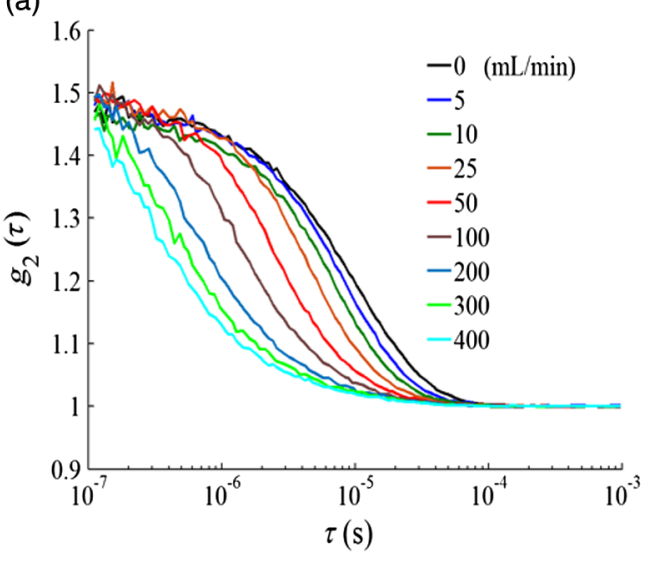

(b)

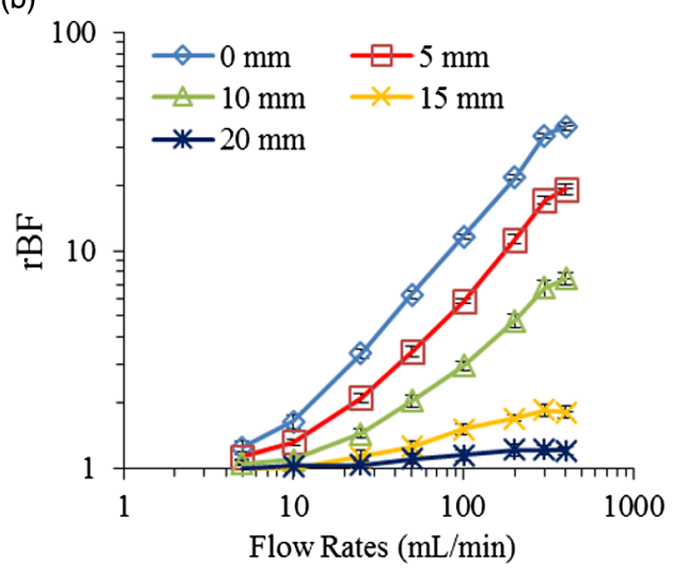

Fig. 2 DCS characterization. (a) Measured intensity autocorrelation functions $g_{2}$, during liquid phantom depth/flow experiment for flow tube $5 \mathrm{~mm}$ below the surface. (b) The relative change of blood flow, rBF, versus pump flow rates, for various flow tube depths. Depth is the distance between the top of the flow tube and the handheld probe. Data are the average of 10 samples, and error bars indicate standard deviation. The DOS-measured optical properties of the liquid phantom were $\mu_{\mathrm{s}}^{\prime}=0.75 \mathrm{~mm}^{-1}$ and $\mu_{\mathrm{a}}=$ $0.005 \mathrm{~mm}^{-1}$ at $785 \mathrm{~nm}$. 


\section{Results}

\subsection{Liquid Phantom Characterization}

The measured intensity autocorrelation functions, $g_{2}$, for different flow rates are shown in Fig. 2(a). The $g_{2}$ exhibits faster decay in the presence of higher flow rate.

Figure 2(b) shows the effect of flow tube depth on rBF for different flow rates. BFI was calculated using the corresponding $\mu_{\mathrm{a}}$ and $\mu_{\mathrm{S}}^{\prime}$ values $\left(0.0048 \mathrm{~mm}^{-1}\right.$ and $0.78 \mathrm{~mm}$, respectively) from DOSI. Compared to a superficial flow tube, the BFI decreased to $52 \%, 24 \%, 10 \%$, and $8 \%$ (mean ratio over all flow rates) when the depth of the tube was $5,10,15$, and $20 \mathrm{~mm}$, respectively.

To illustrate the effect of absorption error on the BFI, two groups of BFI were calculated while varying the background IL mixture $\mu_{\mathrm{a}}$ with a constant flow rate. The first group was calculated by assuming constant $\mu_{\mathrm{a}}=0.0093 \mathrm{~mm}^{-1}$ (median value), and the second group was calculated by using the extracted $\mu_{\mathrm{a}}$ value from DOSI at $785 \mathrm{~nm}$ in each step. The average BFI for each group is shown in Fig. 3(a). BFI measured with constant $\mu_{\mathrm{a}}$ value dropped slightly for a constant flow rate during the absorption titration. However, BFI recovered using DOSI-derived values remained nearly constant (with less than 5\% variation). We observed that a $100 \%$ increase in $\mu_{\mathrm{a}}$ (from 0.0076 to $0.015 \mathrm{~mm}^{-1}$ ) induced almost $20 \%$ range of error on reconstructed BFI. The DOSI-measured $\mu_{\mathrm{s}}^{\prime}(\mathrm{M} \pm \mathrm{SD})$ over the absorption titration was $0.74 \pm 0.031 \mathrm{~mm}^{-1}$. Increased variation in BFI was noted at higher absorption cases due to the decreased DCS count rate and signal-to-noise ratio.

Next, the background IL concentration and thus $\mu_{\mathrm{s}}^{\prime}$ were varied with a constant flow tube depth and rate, with a constant absorption $(\mathrm{M} \pm \mathrm{SD})$ of $0.0041 \pm 0.00027 \mathrm{~mm}^{-1}$. Similar to the absorption test, two groups of BFIs were measured either by assuming $\mu_{\mathrm{s}}^{\prime}$ was constant $\left(0.8 \mathrm{~mm}^{-1}\right)$ or by using the DOSImeasured $\mu_{\mathrm{s}}^{\prime}$. Illustrated in Fig. 3(b), a 60\% increase of $\mu_{\mathrm{s}}^{\prime}$ (from 0.59 to $0.94 \mathrm{~mm}^{-1}$ ) caused a $53 \%$ range of error in BFIsignificantly more than the same proportional change in absorption. When using DOSI-correction, BFI was more consistent (with less than $12 \%$ variation). In general, we observed that an uncertainty of $0.3 \mathrm{~mm}^{-1}$ in $\mu_{\mathrm{s}}^{\prime}$ leads to $\sim 53 \%$ error in BFI, while an uncertainty of $0.003 \mathrm{~mm}^{-1}$ in $\mu_{\mathrm{a}}$ yields only $9 \%$ error.

\subsection{Silicone Phantom}

A phantom with an embedded flow channel was made to demonstrate imaging with the combined probe. The measured optical properties of the phantom (outside of the flow channel) were $\mu_{\mathrm{s}}^{\prime}=0.8 \mathrm{~mm}^{-1}$ and $\mu_{\mathrm{a}}=0.008 \mathrm{~mm}^{-1}$ at $785 \mathrm{~nm}$. DOSI and DCS images calculated from two scans (no flow and $200 \mathrm{~mL} / \mathrm{min}$ flow) are shown in Fig. 4. The values of $\mu_{\mathrm{s}}^{\prime}$ and $\mu_{\mathrm{a}}(\mathrm{M} \pm \mathrm{SD})$ for the three vertical lines (total of 19 before interpolation) centered in the tube were $0.88 \pm 0.0098 \mathrm{~mm}^{-1}$ and $0.010 \pm 0.00097 \mathrm{~mm}^{-1}$, respectively. BFI values $(\mathrm{M} \pm \mathrm{SD})$ in this area were $4.42 \times 10^{-6} \pm 2.52 \times 10^{-6} \mathrm{~mm}^{2} / \mathrm{s}$ for flow $200 \mathrm{~mL} / \mathrm{min}$ and $3.22 \times 10^{-6} \pm 3.19 \times 10^{-7} \mathrm{~mm}^{2} / \mathrm{s}$ for flow $=0 \mathrm{~mL} / \mathrm{min}$.

\subsection{Human Breast Tumor Imaging}

DOSI and DCS images were acquired on a human breast tumor in vivo. The DOSI-measured optical properties over the tumor and surrounding normal tissue are shown in Figs. 5(a) and 5(b). DCS data were collected in a smaller region over the tumor, and images of BFI were calculated using DOSI measured optical properties [Fig. 5(c)] and using constant normal premenopausal breast optical property values $\left(\mu_{\mathrm{a}}=0.005 \mathrm{~mm}^{-1}\right.$ and $\mu_{\mathrm{s}}^{\prime}=$ $0.8 \mathrm{~mm}^{-1}$ ) used in a prior DCS study ${ }^{20}$ [Fig. 5(d)]. Peak contrasts in these maps [Figs. 5(c) and 5(d)] were measured by normalizing the max BFI on the tumor region to the BFI over a normal region (average of three BFI values far from the tumor). The BFI reconstructed with DOSI measured optical properties had a $50 \%$ higher peak contrast than BFI reconstructed using constant optical properties values (267\% versus $217 \%$ ). Figures 6(a)-6(e) show images of breast tissue chromophore concentrations $\left(\mathrm{HbO}_{2}, \mathrm{HbR}\right.$, water, lipid, and TOI) calculated from DOSI-measured absorption. $\mathrm{rMMRO}_{2(\mathrm{~T} / \mathrm{N})} \mathrm{cal}-$ culated from the DOSI-measured $\mathrm{HbO}_{2}$ and $\mathrm{HbR}$ and the BFI is shown in Fig. 6(f). (a)

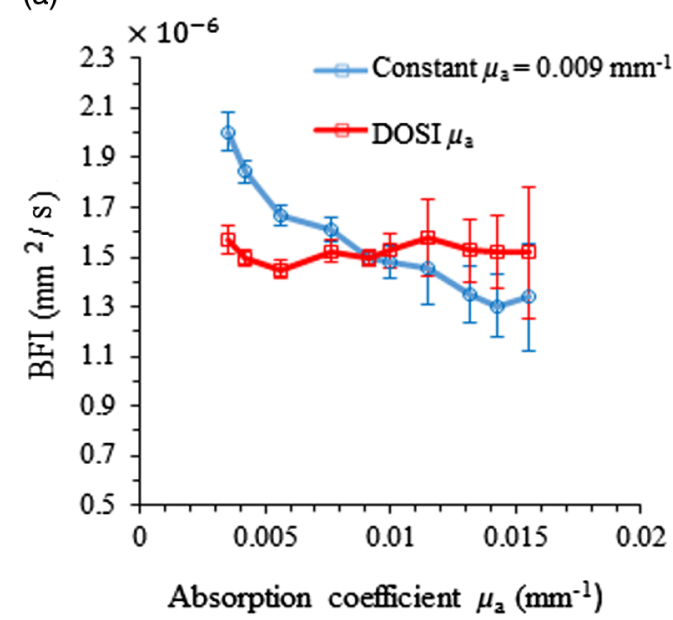

(b)

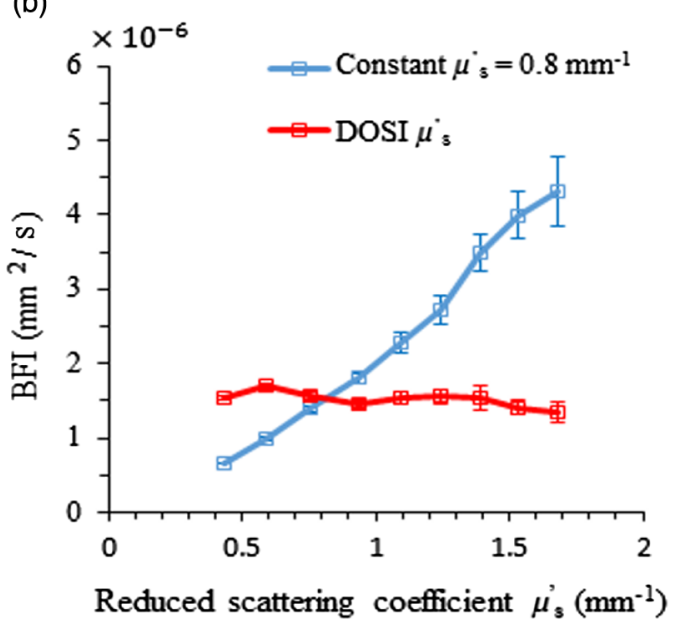

Fig. 3 Effect of absorption and scattering on BFI. BFI calculated with DOSI-corrected and constant optical properties are shown for (a) constant scattering and varied absorption and (b) constant absorption and varied scattering. The flow rate was constant during the measurements. 
(a)

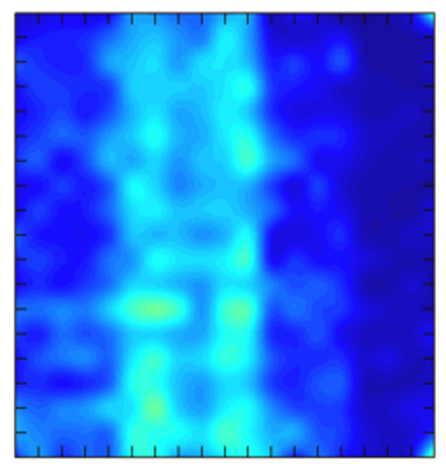

(c)

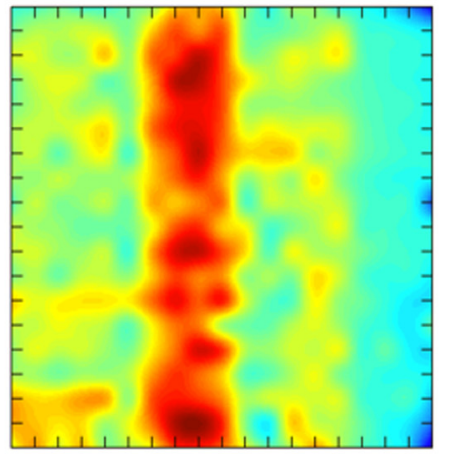

(e)

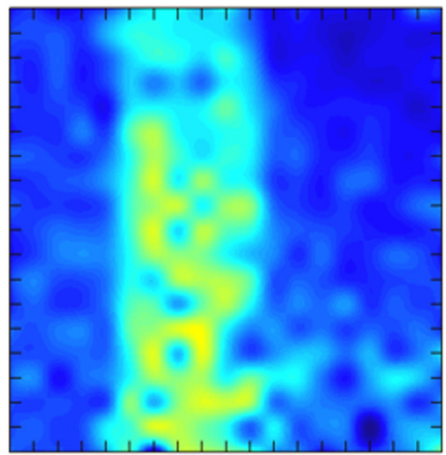

(b)

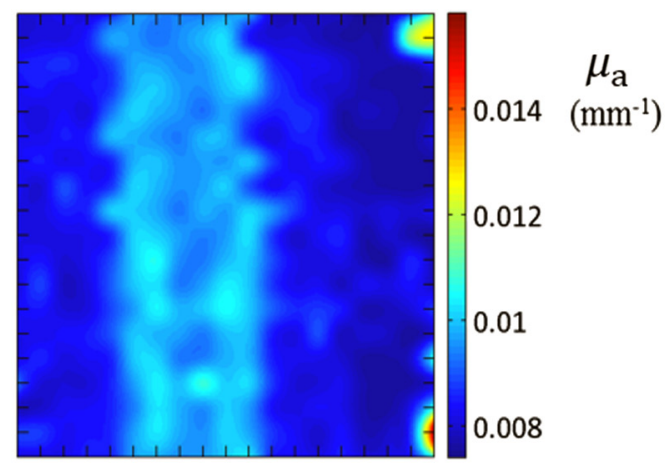

(d)

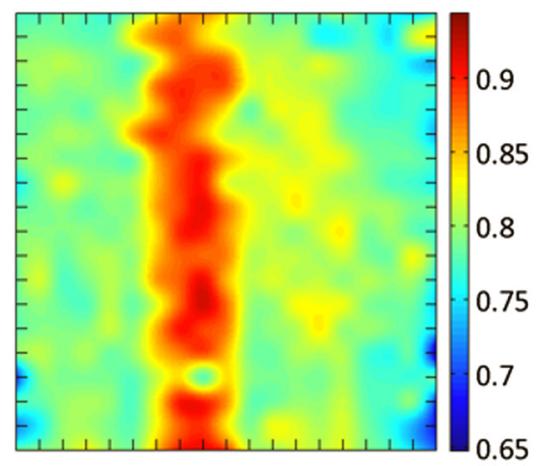

(f)

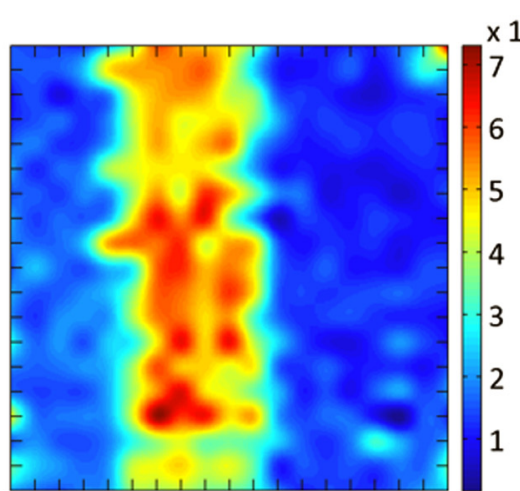

$\mu_{\mathrm{s}}^{\prime}$

$\left(\mathrm{mm}^{-1}\right)$
0.75

.65

BFI

$\left(\mathrm{mm}^{2} / \mathrm{s}\right)$

Fig. 4 DOSI and DCS images of silicone phantom with an embedded flow channel. (a, b) Absorption coefficient $\mu_{\mathrm{a}}$, (c, d) reduced scattering coefficient $\mu_{\mathrm{s}}^{\prime}$, and (e, f) BFI. Images on left represent no flow (a, c, and e), where the images on the right represent, flow (b, $d$, and f). Tick mark separation equals $5 \mathrm{~mm}$.

(a)

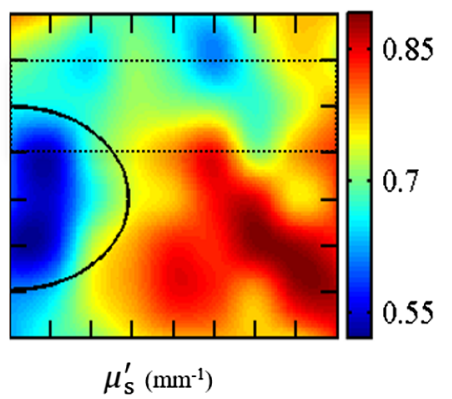

(b)

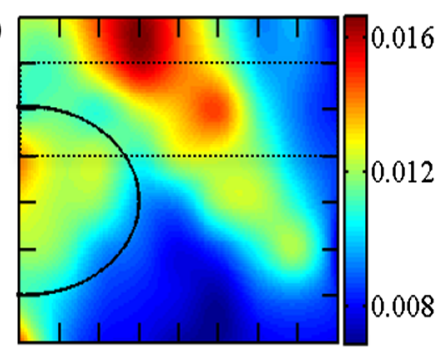

$\mu_{\mathrm{a}}\left(\mathrm{mm}^{-1}\right)$

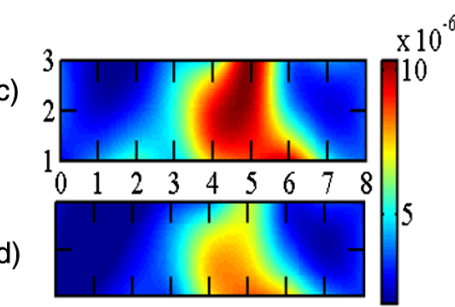

$\operatorname{BFI}\left(\mathrm{mm}^{2} / \mathrm{s}\right)$

Fig. 5 Integrated DOSI and DCS imaging during the clinical measurement of a breast cancer patient (a) $\mu_{\mathrm{s}}^{\prime}$ and (b) $\mu_{\mathrm{a}} \mathrm{mm}^{-1}$ at $785 \mathrm{~nm}$, (c) BFI $\left(\mathrm{mm}^{2} / \mathrm{s}\right)$ reconstructed with $\mu_{\mathrm{s}}^{\prime}$ and $\mu_{\mathrm{a}}$ values measured by DOSI at $785 \mathrm{~nm}$ in each, (d) BFI $\left(\mathrm{mm}^{2} / \mathrm{s}\right)$ calculated with constant $\mu_{\mathrm{s}}^{\prime}$ and $\mu_{\mathrm{a}}$ values $(0.8$ and $0.005 \mathrm{~mm}^{-1}$, respectively). Dashed lines ( $a$ and $b$ ) represent the region where the lesion was located and DOSI/DCS data were taken. The areola region is outlined with a semicircle. Tick marks represent $10 \mathrm{~mm}$ separations. 
(a)

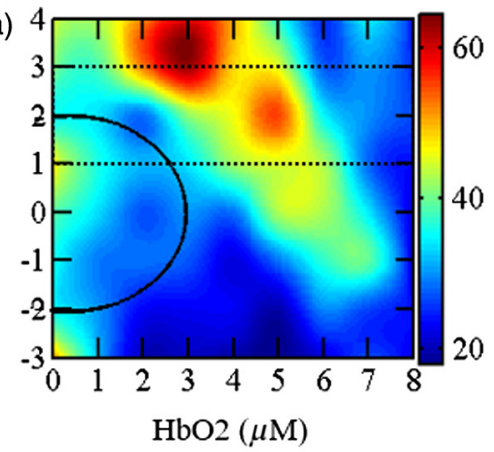

(d)

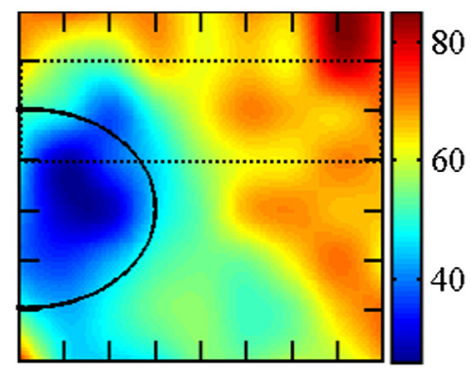

Lipid (\%) (b)

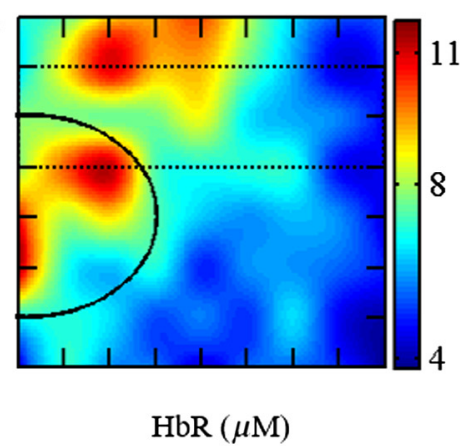

(e)

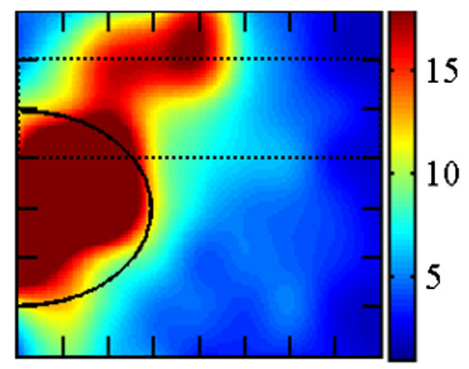

TOI (c)

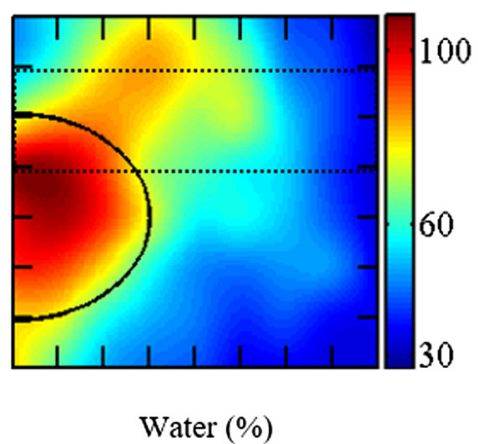

(f)

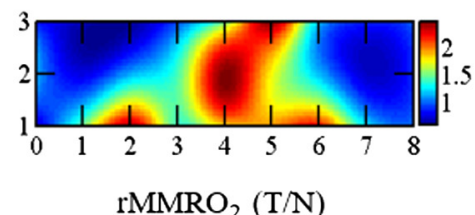

(g)

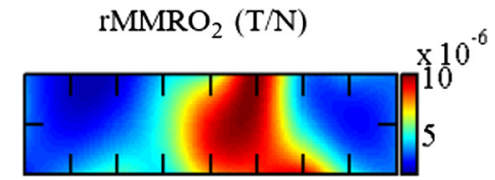

$\mathrm{BFI}\left(\mathrm{mm}^{2} / \mathrm{s}\right)$

Fig. 6 Integrated DOSI and DCS imaging during the clinical measurement of a breast cancer patient. (a) $\mathrm{HbO}_{2}(\mu \mathrm{M})$, (b) $\mathrm{HbR}(\mu \mathrm{M})$, (c) water (\%), (d) lipid (\%), (e) tissue optical index (TOI = $[\mathrm{HbR}] \times[$ water $] /[$ lipid] $),(f) \mathrm{rMMRO}_{2}(T / N)$, and $(\mathrm{g}) \mathrm{BFI}\left(\mathrm{mm}^{2} / \mathrm{s}\right)$. Dashed lines (a-e) represent the region where the lesion was located and DOSI/DCS data were taken. The areola region is outlined with a semicircle. (f and $g$ ) The region that DOSI/DCS data were collected. Tick marks represent $10 \mathrm{~mm}$ separations.

For convenient comparison, the BFI from the same region is shown in Fig. 6(g). We observed that the spatial distributions of $\mathrm{BFI}$ and $\mathrm{rMMRO}_{2(T / N)}$ around the tumor share similar features. However, the spatial distributions of TOI and $\mathrm{rMMRO}_{2(\mathrm{~T} / \mathrm{N})}$ do not match, but the contrast from the areola is apparent in both images.

\section{Discussion}

In this study, we fabricated a combined DOSI/DCS metabolic imaging system designed specifically for breast tissue characterization, validated performance in several phantom studies, and performed breast cancer measurements in a human subject. A single integrated handheld probe was used to collect coregistered DOSI and DCS data from nearly identical tissue regions. Usability and data collection time were optimized by creating a custom software package that allowed the operator to enable either or both optical techniques.

The system was characterized and validated in several liquid and solid phantom experiments. First, a liquid phantom system with a submerged flow tube was developed to evaluate the impact of tube depth, flow speed, and background liquid optical properties on BFI. To simulate the DCS signals relevant to breast tissue, flow rates were selected to yield $\mathrm{rBF}$ (tumor/normal) values (from 1.90 to 2.70 ) at a tube located $15 \mathrm{~mm}$ below the surface based on previously measured flow changes of 32 patients. ${ }^{20}$ Increasing the depth of the flow tube not only decreases the measured flow rate but also reduces flow rate sensitivity and dynamic range. We found that with $28 \mathrm{~mm} \mathrm{~s}-\mathrm{d} \mathrm{sep}-$ aration, a linear flow signal was accessible up to $15 \mathrm{~mm}$ deep. However, while flow changes were accessible for a 20-mm deep flow tube, its linear dynamic range was greatly reduced. $R^{2}$ values derived from linear regression analysis of BFI at depths of $0,5,10,15$, and $20 \mathrm{~mm}$ were $0.98,0.98,0.98,0.86$, and 0.76 , respectively.

Next, we separately varied the $\mu_{\mathrm{a}}$ and $\mu_{\mathrm{s}}^{\prime}$ of the background liquid to demonstrate how bulk tissue optical properties can affect the DCS assessment of BFI. In previously published work, ${ }^{26}$ a homogeneous liquid phantom was used to illustrate the effect of $\mu_{\mathrm{a}}$ and $\mu_{\mathrm{s}}^{\prime}$ on BFI by comparing these values with Brownian motion of particles. In this work, we examined the impact of $\mu_{\mathrm{a}}$ and $\mu_{\mathrm{s}}^{\prime}$ on BFI using a liquid phantom system with a submerged flow tube. Our experiments show that the BFI error is significantly higher for inaccuracies in $\mu_{\mathrm{s}}^{\prime}$ compared to $\mu_{\mathrm{a}}$, and it is independent of the flow rate. Our results are comparable to those from a previous study that showed an uncertainty of $0.3 \mathrm{~mm}^{-1}$ in reduced scattering leads to $\sim 50 \%$ error in BFI, while an uncertainty of $0.003 \mathrm{~mm}^{-1}$ in absorption yields less than $10 \%$ error in BFI at $785 \mathrm{~nm} \cdot{ }^{26}$ Although our absorption and scattering values were chosen to simulate breast tissue, they were varied over a large enough range to provide insight on DCS performance in other tissue types such as muscle and brain. Several phantom studies have investigated BFI error in cerebral blood flow measurements, ${ }^{38,39}$ and they have improved BFI accuracy through more complex modeling that accounts for irregular tissue geometries. ${ }^{40,41}$ It is possible that BFI accuracy in breast measurements can be further improved using models that account for arbitrary geometries ${ }^{42}$ and tissue heterogeneity. ${ }^{43}$ These factors should be addressed in future studies.

A solid tissue-simulating silicone phantom with an embedded flow channel was fabricated for the first time to demonstrate the spatially coregistered information content of $\mu_{\mathrm{a}}, \mu_{\mathrm{s}}^{\prime}$, and BFI. In the region over the flow tube, we observed the expected 
increase in contrast in absorption, scattering, and BFI in both flow and no-flow conditions. BFI contrast in the no-flow case was likely due to the Brownian motion of the liquid scatterers. From these measurements, it is clear that the system is mainly sensitive to deep tissue lying within the field of view defined by the source-detector separation. Limited information is obtained from deep tissue more than $5 \mathrm{~mm}$ laterally outside the sourcedetector plane. Interestingly, the maximum absorption contrast was observed when either the source or detector was positioned directly over the phantom, while the maximum scattering contrast occurred at the midpoint of the source and detector, observations that were confirmed by Monte Carlo simulations (data not presented). Finally, even though the phantom was designed with a centered flow tube, we note that the flow channel appears shifted to the left in all the images. We verified that this is because the reference point used to position the probe was not located at the exact midpoint between the optical sources and detectors.

The combined DOSI/DCS system was used to characterize a high-grade breast tumor in a young patient. Two different images of BFI were calculated by using constant $\mu_{\mathrm{a}}$ and $\mu_{\mathrm{s}}^{\prime}$ values and by extracting these values from the coregistered DOSI data. Although both BFI images showed increased blood flow in the tumor region (slightly different spatial variation), the BFI images reconstructed with DOSI $\mu_{\mathrm{a}}$ and $\mu_{\mathrm{s}}^{\prime}$ exhibited higher BFI. This is due to overestimation of $\mu_{\mathrm{s}}^{\prime}$ (without DOSI) in some tissue regions leading to an underestimation of BFI. Overall, DOSI derived $\mu_{\mathrm{a}}$ and $\mu_{\mathrm{s}}^{\prime}$ can vary up to $100 \%$ and $60 \%$ over different tissue regions, respectively, and the maximum error in the BFI reconstructed with constant $\mu_{\mathrm{a}}$ and $\mu_{\mathrm{s}}^{\prime}$ values ranges from $20 \%$ to $53 \%$. These findings underscore the importance of determining tissue optical and DCS properties from the same location. A prior study indicated that fixed optical properties did not significantly affect the relative change of BFI in tumor versus normal regions in a group of cancer patients. However, individual relative changes in BFI were clearly impacted when using DOSImeasured optical properties. ${ }^{20}$ In addition, assuming constant optical properties can be particularly impactful when monitoring a subject during chemotherapy, since tumor optical properties are changing differently from the background. ${ }^{5}$

Our clinical data further underscore the importance of incorporating blood flow in characterizing breast tumors. Figure 6(f) shows the spatial distribution of oxygen metabolism in and around the tumor compared to the TOI, a high-contrast function used to localize tumors. ${ }^{29}$ We observe colocalized contrast in the images due to the metabolically active tissue of the areola. However, both the peak locations and distributions of TOI and $\mathrm{rMMRO}_{2(T / N)}$ around the tumor do not match. For example, one region of the tumor indicated by increased TOI contrast $[x, y=2$ to $3 \mathrm{~cm}$ in Fig. 6(e)] corresponds to a relatively low $\mathrm{BFI}$ and $\mathrm{rMMRO}_{2(T / N)}$. This is consistent with an increased vascular density but a lower blood flow that results in poor tissue perfusion in this region of the tumor. Consequently, there is a region adjacent to the peak TOI with a high BFI and $\mathrm{rMMRO}_{2(T / N)}$, which may be due to the increased perfusion of peripheral tumor tissue. Overall, these data show how blood flow information augments and complements compositional data to provide a more complete picture of tumor perfusion and metabolism.

\section{Conclusion}

We describe a clinically compatible optical imaging system that simultaneously collects tissue composition and blood flow data using DOSI and DCS, respectively. Specifically, the system measures tissue concentrations of oxy- and deoxyhemoglobin, water, and lipid, as well as blood flow. We validated instrument performance in both liquid and solid phantom systems and demonstrated coregistered imaging in phantoms. Measurements in a patient with breast cancer confirm the importance of accounting for spatial variations in tumor $(T)$ and normal $(N)$ tissue optical properties for optimizing BFI sensitivity and contrast. Finally, hemoglobin and flow parameters were combined to create images of the $T / N$ relative metabolic rate of oxygen consumption $\left[\mathrm{rMMRO}_{2}(T / N)\right]$. Together, quantitative DOSI and DCS imaging provides a more complete view of tumor composition and metabolism, which may aid in predicting, guiding, and personalizing cancer therapies.

\section{Disclosures}

B. J. Tromberg reports patents, which are owned by the University of California and licensed to commercial entities, that are related to the technology and analysis methods described in this study. This research was completed without their participation, knowledge, or financial support, and data were acquired and processed from patients by coauthors unaffiliated with any commercial entity.

\section{Acknowledgments}

This research was supported by the National Institute of Biomedical Imaging and Bioengineering (P41EB015890), the National Cancer Institute (R01CA142989, U54CA136400), and the Chao Family Comprehensive Cancer Center (P30CA62203), with programmatic support from Arnold and Mabel Beckman Foundation. T.S. was supported by a Fulbright Visiting Scholar 2015-6 grant and a Marie Curie and VINNMER Fellow Grant from The Swedish Governmental Agency for Innovation Systems (VINNOVA, 2015-0153). A.G.Y. was partially supported by NIH through P41-EB015893 and 1R01NS060653.

\section{References}

1. A. Yodh and B. Chance, "Spectroscopy and imaging with diffusing light," Phys. Today 48(3), 34-40 (1995).

2. A. Leproux et al., "Assessing tumor contrast in radiographically dense breast tissue using diffuse optical spectroscopic imaging (DOSI)," Breast Cancer Res. 15(5), R89 (2013).

3. S. Kukreti et al., "Characterization of metabolic differences between benign and malignant tumors: high-spectral-resolution diffuse optical spectroscopy," Radiology 254(1), 277-284 (2010).

4. A. E. Cerussi et al., "Frequent optical imaging during breast cancer neoadjuvant chemotherapy reveals dynamic tumor physiology in an individual patient," Acad. Radiol. 17(8), 1031-1039 (2010).

5. D. Roblyer et al., "Optical imaging of breast cancer oxyhemoglobin flare correlates with neoadjuvant chemotherapy response one day after starting treatment," Proc. Natl. Acad. Sci. U. S. A. 108(35), 14626 (2011).

6. A. E. Cerussi et al., "Diffuse optical spectroscopic imaging correlates with final pathological response in breast cancer neoadjuvant chemotherapy," Philos. Trans. R. Soc. London A Math. Phys. Eng. Sci. 369(1955), 4512-4530 (2011).

7. T. D. O'sullivan et al., "Optical imaging correlates with magnetic resonance imaging breast density and reveals composition changes during neoadjuvant chemotherapy," Breast Cancer Res. 15(1), R14 (2013).

8. D. Boas, L. Campbell, and A. Yodh, "Scattering and imaging with diffusing temporal field correlations," Phys. Rev. Lett. 75(9), 1855-1858 (1995).

9. D. Boas and A. Yodh, "Spatially varying dynamical properties of turbid media probed with diffusing temporal light correlation," J. Opt. Soc. Am. A 14(1), 192-215 (1997). 
10. T. Durduran et al., "Diffuse optical measurement of blood flow in breast tumors," Opt. Lett. 30(21), 2915-2917 (2005).

11. G. Yu et al., "Validation of diffuse correlation spectroscopy for muscle blood flow with concurrent arterial spin labeled perfusion MRI," Opt. Exp. 15(3), 1064-1075 (2007).

12. E. M. Buckley et al., "Cerebral hemodynamics in preterm infants during positional intervention measured with diffuse correlation spectroscopy and transcranial Doppler ultrasound," Opt. Express 17(15), 12571 (2009).

13. N. Roche-Labarbe et al., "Noninvasive optical measures of $\mathrm{CBV}, \mathrm{StO}_{2}$, $\mathrm{CBF}$ index, and $\mathrm{rCMRO}_{2}$ in human premature neonates' brains in the first six weeks of life," Hum. Brain Mapp. 31(3), 341-352 (2010).

14. M. N. Kim et al., "Noninvasive measurement of cerebral blood flow and blood oxygenation using near-infrared and diffuse correlation spectroscopies in critically brain-injured adults," Neurocrit. Care 12(2), 173180 (2010)

15. T. Durduran, "Non-invasive measurements of tissue hemodynamics with hybrid diffuse optical methods," Med. Phys. 31(7), 2178 (2004).

16. Y. Shang et al., "Diffuse optical monitoring of repeated cerebral ischemia in mice," Opt. Express 19(21), 20301 (2011).

17. R. Cheng et al., "Noninvasive optical evaluation of spontaneous low frequency oscillations in cerebral hemodynamics," Neuroimage 62(3), 1445-1454 (2012).

18. G. Yu et al., "Time-dependent blood flow and oxygenation in human skeletal muscles measured with noninvasive near-infrared diffuse optical spectroscopies," J. Biomed. Opt. 10(2), 024027 (2005).

19. G. Yu et al., "Intraoperative evaluation of revascularization effect on ischemic muscle hemodynamics using near-infrared diffuse optical spectroscopies," J. Biomed. Opt. 16(2), 027004 (2011).

20. R. Choe et al., "Optically measured microvascular blood flow contrast of malignant breast tumors," PloS One 9(6), e99683 (2014).

21. L. Dong et al., "Noninvasive diffuse optical monitoring of head and neck tumor blood flow and oxygenation during radiation delivery," Biomed. Opt. Express 3(2), 259-272 (2012).

22. U. Sunar et al., "Noninvasive diffuse optical measurement of blood flow and blood oxygenation for monitoring radiation therapy in patients with head and neck tumors: a pilot study," J. Biomed. Opt. 11(6), 064021 (2006).

23. C. Zhou et al., "Diffuse optical monitoring of blood flow and oxygenation in human breast cancer during early stages of neoadjuvant chemotherapy," J. Biomed. Opt. 12(5), 051903 (2007).

24. Y. Shang et al., "Portable optical tissue flow oximeter based on diffuse correlation spectroscopy," Opt. Lett. 34(22), 3556-3558 (2009).

25. D. Jakubowski et al., "Quantitative absorption and scattering spectra in thick tissues using broadband diffuse optical spectroscopy," in Biomedical Optical Imaging, J. Fujimoto and D. Farkas, Eds., pp. 330-355, Oxford University Press, New York (2009).

26. D. Irwin et al., "Influences of tissue absorption and scattering on diffuse correlation spectroscopy blood flow measurements," Biomed. Opt. Express 2(7), 1969-1985 (2011).

27. G. Maret and P. Wolf, "Multiple light scattering from disordered media. The effect of Brownian motion of scatterers," Zeitschrift für Physik B Condens. Matter 65(4), 409-413 (1987).

28. D. Pine et al., "Diffusing wave spectroscopy," Phys. Rev. Lett. 60(12), 1134-1137 (1988).

29. S. Rice, Selected Topics in Noise and Stochastic Processes, Dover, New York (1954)

30. D. A. Boas et al., "Near-infrared diffuse correlation spectroscopy for assessment of tissue blood flow," Handbook of Biomedical Optics, pp. 195-216, CRC Press, Boca Raton (2011).

31. D. A. Boas, "Diffuse photon probes of structural and dynamical properties of turbid media: theory and biomedical applications," $\mathrm{PhD}$ Dissertation, University of Pennsylvania, Philadelphia (1996).

32. X. Li, "Fluorescence and diffusive wave diffraction tomographic probes in turbid media," PhD Dissertation, University of Pennsylvania, Philadelphia (1998).

33. C. Zhou, "In-vivo optical imaging and spectroscopy of cerebral hemodynamics," PhD Dissertation, University of Pennsylvania, Philadelphia (2007).

34. S. A. Walker, S. Fantini, and E. Gratton, "Effect of index of refraction mismatch on the recovery of optical properties of cylindrical inhomogeneities in an infinite turbid medium," Proc. SPIE 2979, 219 (1997).
35. C. Cheung et al., "In vivo cerebrovascular measurement combining diffuse near-infrared absorption and correlation spectroscopies," Phys. Med. Biol. 46(8), 2053-2065 (2001).

36. T. D. O'Sullivan et al., "Diffuse optical imaging using spatially and temporally modulated light," J. Biomed. Opt. 17(7), 071311 (2012).

37. K.-S. No et al., "Design and testing of a miniature broadband frequency domain photon migration instrument," J. Biomed. Opt. 13(5), 050509 (2008).

38. J. Selb et al., "Sensitivity of near-infrared spectroscopy and diffuse correlation spectroscopy to brain hemodynamics: simulations and experimental findings during hypercapnia," Neurophotonics 1(1), 015005 (2014).

39. K. Verdecchia et al., Eds., "Characterization of a hybrid diffuse correlation spectroscopy and time-resolved near-infrared spectroscopy system for real-time monitoring of cerebral blood flow and oxygenation," Proc. SPIE 9313, 931310 (2015).

40. L. Gagnon et al., "Investigation of diffuse correlation spectroscopy in multi-layered media including the human head," Opt. Express 16(20), 15514 (2008).

41. K. Verdecchia et al., "Assessment of a multi-layered diffuse correlation spectroscopy method for monitoring cerebral blood flow in adults," Biomed. Opt. Express 7(9), 3659-3674 (2016).

42. Y. Shang et al., "Extraction of diffuse correlation spectroscopy flow index by integration of $\mathrm{N}$ th-order linear model with Monte Carlo simulation," Appl. Phys. Lett. 104(19), 193703 (2014).

43. Y. Shang and G. Yu, "AN th-order linear algorithm for extracting diffuse correlation spectroscopy blood flow indices in heterogeneous tissues," Appl. Phys. Lett. 105(13), 133702 (2014).

Hossein S. Yazdi is a graduate student researcher at the Beckman Laser Institute and Medical Clinic, University of California, Irvine under supervision of Dr. Bruce Tromberg. He received his BSc degree in electrical engineering from Azad University, Iran, and his MSc degree in biomedical engineering from Linkoping University, Sweden, in 2013. He is currently working on a multimodal technique combining diffuse optical spectroscopic imaging and diffuse correlation spectroscopy.

Thomas D. O'Sullivan is an assistant professor in the Department of Electrical Engineering at the University of Notre Dame. He received his $\mathrm{PhD}$ in electrical engineering from Stanford University in 2011 and then was a U.S. Department of Defense Breast Cancer Research Program postdoctoral fellow at the University of California, Irvine Beckman Laser Institute (BLI). While at BLI, he also served as a director of the diffuse optical spectroscopy and imaging laboratory.

Anais Leproux received her PhD in biomedical engineering in 2012 at the University of Amsterdam, Netherlands. She has been working as a research scientist at the Beckman Laser Institute (BLI) since then. Her BLI research in biomedical photonics is focused on using diffuse optical spectroscopic imaging (DOSI) technology in breast cancer applications, mainly for the detection and characterization of breast tumor response to therapy.

Jesse Lam is a second-year PhD student in the Department of Biomedical Engineering at the University of California, Irvine, where he also earned his bachelor's degree in biomedical engineering. He is currently a graduate student researcher at the Beckman Laser Institute and Medical Clinic in Dr. Tromberg's lab, where he studies diffuse optical spectroscopy and imaging.

Siavash S. Yazdi received his BS degree in electrical engineering from Sharif University of Technology, Tehran, Iran, in 2011. At the same year, he started his graduate studies at the University of California, Irvine, California, where he is currently working toward his $\mathrm{PhD}$ in electrical engineering. His current research interests are high-speed analog and mixed signal circuits for biomedicine and communications.

Robert M. Carroll is an associate clinical professor in the $\mathrm{UCl}$ Division of Hematology and Medical Oncology. He completed his BS degree in biochemistry at the UC San Diego and his Doctor of Medicine at Hahnemann University. His residency in internal medicine and fellowship in hematology and medical oncology at Harbor-UCLA Medical Center. He has been faculty at UC Irvine 
since 2013 and treats wide range of malignancies with a special focus in breast cancer.

Tomas Strömberg is a professor and head of the Department of Biomedical Engineering at Linköping University. His current research interests are within biomedical optics, especially pointwise and imaging laser Doppler flowmetry, laser speckle contrast analysis, diffuse reflectance spectroscopy, and Monte Carlo simulations. Applications are within microcirculation characterization in diabetes and peripheral vascular disease.

Arjun G. Yodh is a Skinner professor of science in the Department of Physics and Astronomy at the University of Pennsylvania. He also directs the Laboratory for Research on the Structure of Matter, an interdisciplinary materials institute that hosts the Penn MRSEC. His biomedical research is oriented toward diffuse optical imaging and monitoring, with aims to demonstrate the potential of these tools for functional imaging/monitoring in brain, breast, and muscle, and for monitoring hemodynamic biomarkers during treatment.

Bruce J. Tromberg is a professor in the Departments of Biomedical Engineering and Surgery and director of the Beckman Laser Institute and Medical Clinic (BLI) at the University of California, Irvine (UCI). He has been a member of the BLI Faculty since 1990. His research focuses on the development of diffuse optical spectroscopy and imaging, nonlinear optical microscopy, and multimodality imaging technologies, with particular emphasis on quantitative imaging endpoints for detecting disease and improving therapeutic outcome for patients.

Biographies for the other authors are not available. 